\title{
Discovery of anomalous oxygen isotopic ratios in HR 4049^
}

\author{
J. Cami ${ }^{1,2}$ and I. Yamamura ${ }^{3}$ \\ 1 Astronomical Institute "Anton Pannekoek", University of Amsterdam, Kruislaan 403, 1098 SJ Amsterdam, \\ The Netherlands \\ 2 SRON-Groningen, PO Box 800, 9700 AV Groningen, The Netherlands \\ 3 The Institute of Space and Astronautical Science (ISAS), Yoshino-dai 3-1-1, Sagamihara, Kanagawa 229-8510, \\ Japan \\ e-mail: yamamura@ir.isas.ac.jp
}

Received 30 November 2000 / Accepted 22 December 2000

\begin{abstract}
We report the discovery in the ISO/SWS spectrum of the post-AGB star HR 4049 of emission bands due to ${ }^{17} \mathrm{O}$ and ${ }^{18} \mathrm{O}$ isotopes locked up in $\mathrm{CO}_{2}$ molecules. It is the first time these isotopomers are detected outside the solar system. Isotopic ratios derived in the optically thin limit are as low as ${ }^{16} \mathrm{O} /{ }^{17} \mathrm{O}=8.3 \pm 2.3$ and ${ }^{16} \mathrm{O} /{ }^{18} \mathrm{O}=6.9 \pm 0.9$. These values are at least one order of magnitude lower than any previously determined isotopic ratio in any type of evolved star.
\end{abstract}

Key words. stars: AGB and post-AGB - stars: circumstellar matter - stars: individual: HR 4049 stars: abundances - stars: late-type - infrared: stars

\section{Introduction}

HR 4049 is considered to be the prototype of a group of post-AGB stars in a binary system with extremely metaldepleted photospheres (van Winckel et al. 1995). The photospheric abundance patterns of these stars are the result of dust formation in a circumbinary disk followed by accretion of the depleted gas (Waters et al. 1992). As the photospheric chemical composition is severely altered by this process, photospheric abundances cannot be used to determine the evolutionary status of HR 4049 and therefore the evolution of this object can only be traced by studying the circumstellar material.

The presence of strong IR emission features attributed to Polycyclic Aromatic Hydrocarbons (PAHs, see Waters et al. 1989; Beintema et al. 1996) suggests that the circumstellar material surrounding HR 4049 is C-rich. Further evidence for C-rich dust is found from studying the profiles of the 3.43 and $3.53 \mu \mathrm{m}$ emission features (Geballe et al. 1989) recently identified with vibrational modes of hydrogen-terminated crystalline facets of diamond (Guillois et al. 1999). However, a search for C-rich

Send offprint requests to: J. Cami,

e-mail: cami@astro.uva.nl

* Based on observations with ISO, an ESA project with instruments funded by ESA Member States (especially the PI countries: France, Germany, the Netherlands and the UK) with the participation of ISAS and NASA. The SWS is a joint project of SRON and MPE. molecules like $\mathrm{C}_{2}, \mathrm{CN}$ and $\mathrm{CH}^{+}$(Bakker et al. 1997) turned out negative.

In this paper we present part of the ISO-SWS lowresolution full-grating spectrum (AOT1, speed 2). The full spectrum and analysis will be discussed in detail in a forthcoming paper (Cami et al. 2001, Paper I hereafter). In this paper we report the detection of several $\mathrm{CO}_{2}$ isotopomers in the $13-17 \mu \mathrm{m}$ spectral region: ${ }^{12} \mathrm{C}^{16} \mathrm{O}_{2}$, ${ }^{13} \mathrm{C}^{16} \mathrm{O}_{2},{ }^{16} \mathrm{O}^{12} \mathrm{C}^{18} \mathrm{O}$ and ${ }^{16} \mathrm{O}^{12} \mathrm{C}^{17} \mathrm{O}$. It is the first time these last two isotopomers are detected outside the solar system.

\section{2. $\mathrm{CO}_{2}$ isotopomers in the $13-17 \mu \mathrm{m}$ region}

It is well known that the spectra of several O-rich AGB stars exhibit a few strong $\mathrm{CO}_{2}$ bands in this wavelength range, most often seen in emission (Cami et al. 1997; Justtanont et al. 1998; Ryde et al. 1998; Cami et al. 2000; Markwick \& Millar 2000). In the spectrum of HR 4049, all previously observed bands are present and in emission; it is obvious from first inspection however that the $\mathrm{CO}_{2}$ spectrum of HR 4049 is much richer in lines than any other object observed so far (see Fig. 1). The strongest emission band in this region is observed at $14.95 \mu \mathrm{m}$ and is due to ${ }^{12} \mathrm{C}^{16} \mathrm{O}_{2}$. As this band is the combination of the fundamental $\nu_{2}$ bending mode at $14.98 \mu \mathrm{m}$ and resonant hot bands which are subsequently shifted to the blue, the peak position of this band depends strongly on the temperature (Cami et al. 2000). The blueshift of the 

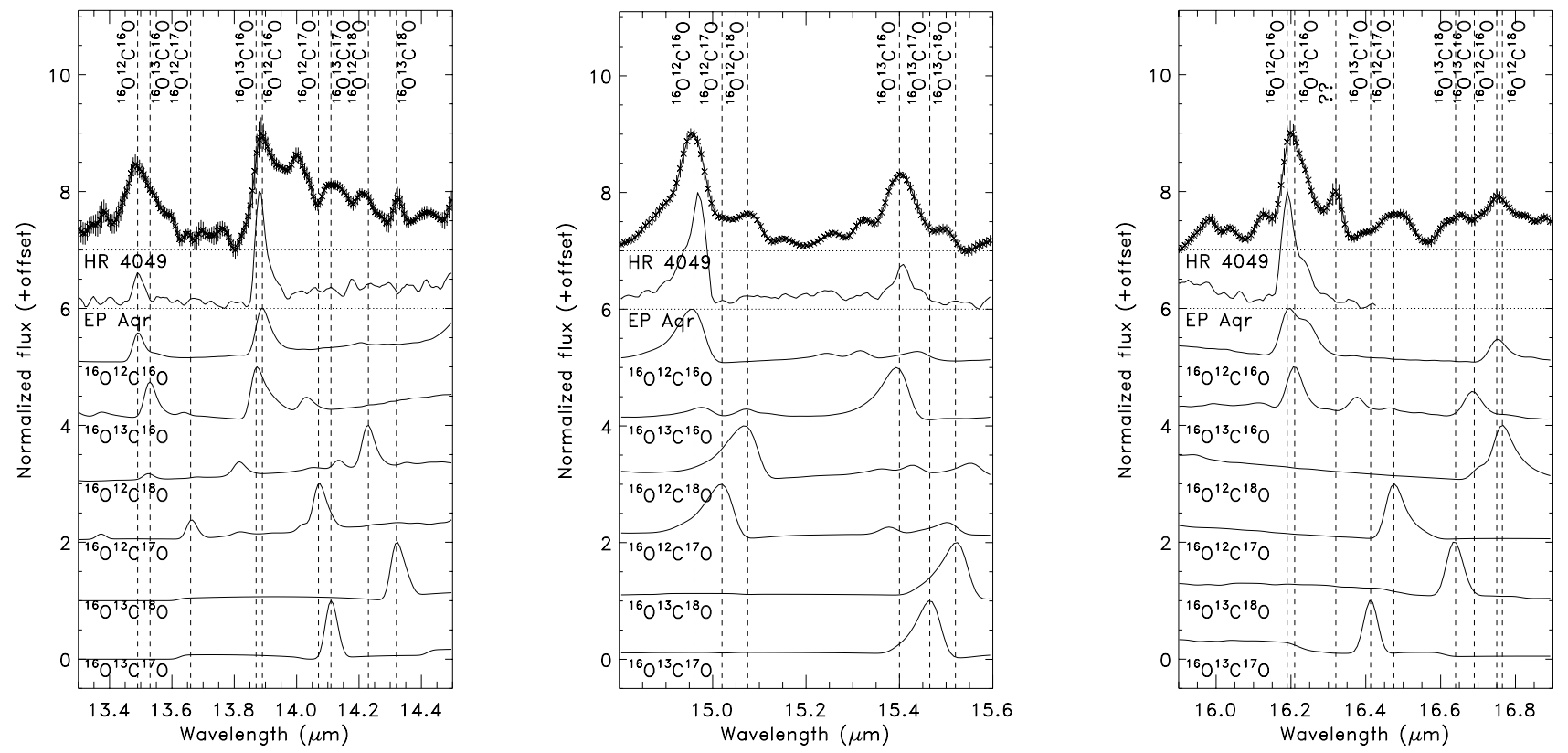

Fig. 1. Parts of the ISO/SWS spectrum of HR 4049 (top curve) between 13-17 $\mu \mathrm{m}$ showing the strongest $\mathrm{CO}_{2}$ emission bands. The spectrum is continuum subtracted and subsequently scaled to the peak flux in each plot window. Error bars on the individual datapoints are indicated. For comparison we also show the spectrum of EP Aqr, rebinned to the same resolution as the spectrum of HR 4049. An LTE model spectrum is then shown for each $\mathrm{CO}_{2}$ isotopomer, with model parameters $T=600 \mathrm{~K}$, $N=10^{19} \mathrm{~cm}^{-2}$. All model spectra are scaled to the peak flux in each plot window and should therefore only be used for identification purposes. The features observed at 14.0 and $14.31 \mu \mathrm{m}$ are close to the expected positions for the two strongest HCN bands in this wavelength range, as observed in carbon stars. However, LTE models of these bands show that the peak positions of both features are off by at least $0.02 \mu \mathrm{m}$ indicating that HCN is not the major constituent of these features

observed peak thus indicates warm gas. ${ }^{12} \mathrm{C}^{16} \mathrm{O}_{2}$ hot bands are at $13.48,13.87$ and $16.18 \mu \mathrm{m}$. The spectrum moreover shows ${ }^{12} \mathrm{C}^{16} \mathrm{O}_{2}$ emission bands which are not previously observed; for instance, an emission feature around $16.75 \mu \mathrm{m}$ can be identified with the $03^{1} 0 \rightarrow 02^{2} 0$ $Q$-branch band.

The ${ }^{13} \mathrm{C}^{16} \mathrm{O}_{2}$ isotopomer shows a strong emission band due to the fundamental $\nu_{2}$ bending mode at $15.42 \mu \mathrm{m}$ and hot bands at 13.52, 13.86 and $16.20 \mu \mathrm{m}$, blended with the corresponding ${ }^{12} \mathrm{C}^{16} \mathrm{O}_{2}$ bands.

When comparing to the spectra of O-rich AGB stars like EP Aqr (Cami et al. 2000), the hot bands observed in the spectrum of HR 4049 are unusually strong compared to the $14.98 \mu \mathrm{m}$ band and much broader, indicating a contribution from other species.

In addition to the bands due to the ${ }^{12} \mathrm{C}^{16} \mathrm{O}_{2}$ and ${ }^{13} \mathrm{C}^{16} \mathrm{O}_{2}$ isotopomers there seem to be other bands present in the 13-17 $\mu \mathrm{m}$ region in the ISO/SWS spectrum of HR 4049. Our attention was caught by a conspicuous peak on the red side of the $14.98 \mu \mathrm{m}$ band. This peak is present in two independent scans of this spectral region, and we verified its presence also in the previously published spectrum (AOT 1 speed 1, see Beintema et al. 1996), confirming that this feature is real. This band peaks at $15.08 \mu \mathrm{m}$ which we identify with the fundamental $\nu_{2}$ bending mode of the ${ }^{16} \mathrm{O}^{12} \mathrm{C}^{18} \mathrm{O}$ isotopomer. The bandheads of the $02^{0} 0$ $\rightarrow 01^{1} 0$ and $10^{0} 0 \rightarrow 01^{1} 0$ bands of this isotopomer should be at 16.75 and $14.21 \mu \mathrm{m}$ respectively. The first one is probably present in the spectrum but coincides with the
$03^{1} 0 \rightarrow 02^{2} 0$ band of ${ }^{12} \mathrm{C}^{16} \mathrm{O}_{2}$; the second one can be clearly seen as an emission feature. The $11^{1} 0 \rightarrow 02^{2} 0$ band of this isotopomer (at $13.81 \mu \mathrm{m}$ ) is not detected.

At $15.04 \mu \mathrm{m}$ there might be emission from the fundamental $\nu_{2}$ bending mode of the ${ }^{16} \mathrm{O}^{12} \mathrm{C}^{17} \mathrm{O}$ isotopomer. The $02^{0} 0 \rightarrow 01^{1} 0$ and $10^{0} 0 \rightarrow 01^{1} 0$ bands of this isotopomer should be at 16.46 and $14.06 \mu \mathrm{m}$; the former is clearly present in the spectrum of HR 4049 as an isolated emission feature while the latter does not exhibit a strong emission but might well be hidden in the broad shoulders from the corresponding ${ }^{12} \mathrm{C}^{16} \mathrm{O}_{2}$ and ${ }^{13} \mathrm{C}^{16} \mathrm{O}_{2}$ bands. The $11^{1} 0 \rightarrow 02^{2} 0$ band of this isotopomer should be at $13.66 \mu \mathrm{m}$ and might be a small wobble in the spectrum.

Given the relative strength of the fundamental ${ }^{12} \mathrm{C}^{16} \mathrm{O}_{2}$ and ${ }^{13} \mathrm{C}^{16} \mathrm{O}_{2}$ bands, and the relative strength of the ${ }^{12} \mathrm{C}^{16} \mathrm{O}_{2}$ and ${ }^{16} \mathrm{O}^{12} \mathrm{C}^{18} \mathrm{O}$ and ${ }^{16} \mathrm{O}^{12} \mathrm{C}^{17} \mathrm{O}$ bands, one might also expect to see (a) band(s) of the ${ }^{16} \mathrm{O}^{13} \mathrm{C}^{18} \mathrm{O}$ and ${ }^{16} \mathrm{O}^{13} \mathrm{C}^{17} \mathrm{O}$ isotopomers. ${ }^{16} \mathrm{O}^{13} \mathrm{C}^{18} \mathrm{O}$ has its fundamental $\nu_{2}$ bending mode at $15.54 \mu \mathrm{m}$ and might be the bump at the red side of the ${ }^{13} \mathrm{C}^{16} \mathrm{O}_{2}$ fundamental. Although the observed peak wavelength seems to be significantly shifted to the blue, we note that for this isotopomer the HITEMP database used for identification of the bands includes only one resonant hot band which is indeed blueshifted with respect to the fundamental. Moreover the observed blueshift for this isotopomer is of the same order as the observed blueshift of the ${ }^{12} \mathrm{C}^{16} \mathrm{O}_{2}$ isotopomer when comparing to the fundamental. We therefore believe that the observed shift is due to high gas temperatures combined with the 
Table 1. Expected wavelengths versus observed peak fluxes of the $Q$-branch bands in the $13-17 \mu \mathrm{m}$ region for the different $\mathrm{CO}_{2}$ isotopomers. The first column lists the vibrational transition considered. For each isotopomer the first column gives the wavelength corresponding to the bandhead of this transition. The second column for each isotopomer gives the observed peak flux (in Jy) if an emission band is clearly present. If the band considered coincides with a band from a more abundant isotope, this is marked with a (B). If there is not a very clear emission feature in the spectrum, but the band might be present in the shoulder of another band, the flux is preceded with a " $\sim$ ". The last band for the two least abundant isotopomers is not listed in HITEMP and therefore marked with a question mark. The uncertainties on the measured peak fluxes are typically $\pm 0.75 \mathrm{Jy}$

\begin{tabular}{|c|c|c|c|c|c|c|c|c|c|c|c|c|}
\hline \multirow[t]{2}{*}{ Band } & \multicolumn{2}{|c|}{${ }^{12} \mathrm{C}^{16} \mathrm{O}_{2}$} & \multicolumn{2}{|c|}{${ }^{13} \mathrm{C}^{16} \mathrm{O}_{2}$} & \multicolumn{2}{|c|}{${ }^{16} \mathrm{O}^{12} \mathrm{C}^{18} \mathrm{O}$} & \multicolumn{2}{|c|}{${ }^{16} \mathrm{O}^{12} \mathrm{C}^{17} \mathrm{O}$} & \multicolumn{2}{|c|}{${ }^{16} \mathrm{O}^{13} \mathrm{C}^{18} \mathrm{O}$} & \multicolumn{2}{|c|}{${ }^{16} \mathrm{O}^{13} \mathrm{C}^{17} \mathrm{O}$} \\
\hline & $\begin{array}{c}\lambda \\
(\mu \mathrm{m}) \\
\end{array}$ & $\begin{array}{c}F_{\nu} \\
(\mathrm{Jy}) \\
\end{array}$ & $\begin{array}{c}\lambda \\
(\mu \mathrm{m}) \\
\end{array}$ & $\begin{array}{c}F_{\nu} \\
(\mathrm{Jy}) \\
\end{array}$ & $\begin{array}{c}\lambda \\
(\mu \mathrm{m}) \\
\end{array}$ & $\begin{array}{c}F_{\nu} \\
(\mathrm{Jy}) \\
\end{array}$ & $\begin{array}{c}\lambda \\
(\mu \mathrm{m}) \\
\end{array}$ & $\begin{array}{c}F_{\nu} \\
(\mathrm{Jy}) \\
\end{array}$ & $\begin{array}{c}\lambda \\
(\mu \mathrm{m}) \\
\end{array}$ & $\begin{array}{c}F_{\nu} \\
(\mathrm{Jy}) \\
\end{array}$ & $\begin{array}{c}\lambda \\
(\mu \mathrm{m}) \\
\end{array}$ & $\begin{array}{c}F_{\nu} \\
(\mathrm{Jy}) \\
\end{array}$ \\
\hline $01^{1} 0 \rightarrow 00^{0} 0$ & 14.98 & 16.8 & 15.42 & 11.0 & 15.10 & 5.5 & 15.04 & $\sim 4.9$ & 15.54 & 3.3 & 15.49 & $\sim 3.0$ \\
\hline $02^{0} 0 \rightarrow 01^{1} 0$ & 16.18 & 9.1 & 16.20 & (B) 9.1 & 16.75 & 4.3 & 16.46 & 2.9 & 16.62 & $\sim 2.9$ & 16.40 & $\sim 1.6$ \\
\hline $10^{0} 0 \rightarrow 01^{1} 0$ & 13.87 & 5.5 & 13.86 & (B) 5.5 & 14.21 & 2.0 & 14.06 & $\sim 1.4$ & 14.31 & 1.7 & 14.10 & 2.5 \\
\hline $11^{1} 0 \rightarrow 02^{2} 0$ & 13.48 & 3.7 & 13.52 & (B) 3.7 & 13.81 & - & 13.66 & $\sim 0.5$ & $?$ & & $?$ & \\
\hline
\end{tabular}

incompleteness of the HITEMP database. The HITEMP database furthermore only lists 2 more bands in this wavelength range for this particular isotopomer, the $02^{0} 0 \rightarrow$ $01^{1} 0$ band at $16.62 \mu \mathrm{m}$ and the $02^{0} 0 \rightarrow 01^{1} 0$ band at $14.31 \mu \mathrm{m}$. The first one is probably blended with the corresponding bands of the other isotopomers; the second one can be clearly seen as an isolated emission feature. The fundamental for the ${ }^{16} \mathrm{O}^{13} \mathrm{C}^{17} \mathrm{O}$ isotopomer seems not to be present at the right wavelength $(15.49 \mu \mathrm{m})$, but also here we would expect the band to be blueshifted and therefore be blended with the ${ }^{13} \mathrm{C}^{16} \mathrm{O}_{2}$ fundamental. The HITEMP database again lists only two more bands, the $02^{0} 0 \rightarrow 01^{1} 0$ band at $16.40 \mu \mathrm{m}$ and the $02^{0} 0 \rightarrow 01^{1} 0$ band at $14.10 \mu \mathrm{m}$. The first one might be a small wobble; a contribution from the second seems to be present in the spectrum in a more or less isolated region.

Table 1 gives a summary of the expected peak positions and the observed peak fluxes for the four strongest bands for each isotopomer.

\section{Oxygen isotopic ratios}

The detection of O-rich molecules in an environment where all the dust seems to be C-rich is a surprise in itself, and will be discussed in detail in Paper I. The detection of ${ }^{17} \mathrm{O}$ and ${ }^{18} \mathrm{O}$ isotopes is even more of a surprise considering predicted and observed isotopic ratios for various types of evolved stars (see Fig. 2).

From the measured line intensities one can estimate isotopic ratios assuming that the lines are optically thin and formed in LTE. When using the line intensities of the ${ }^{12} \mathrm{C}^{16} \mathrm{O}_{2}$ and ${ }^{16} \mathrm{O}^{12} \mathrm{C}^{17} \mathrm{O}$ bands for instance, one finds

$$
\begin{aligned}
\frac{{ }^{16} \mathrm{O}}{{ }^{17} \mathrm{O}} & =\frac{2 \times \mathcal{N}^{12} \mathrm{C}^{16} \mathrm{O}_{2}+\mathcal{N}^{16} \mathrm{O}^{12} \mathrm{C}^{17} \mathrm{O}}{\mathcal{N}^{16} \mathrm{O}^{12} \mathrm{C}^{17} \mathrm{O}} \\
& =2 \frac{\mathcal{N}^{12} \mathrm{C}^{16} \mathrm{O}_{2}}{\mathcal{N}^{16} \mathrm{O}^{12} \mathrm{C}^{17} \mathrm{O}}+1 .
\end{aligned}
$$

In the optically thin limit the line intensity of a transition at frequency $\nu$ between an upper level $u$ and a lower level $l$ scales with the number of excited molecules while in LTE the Boltzmann equation relates the number

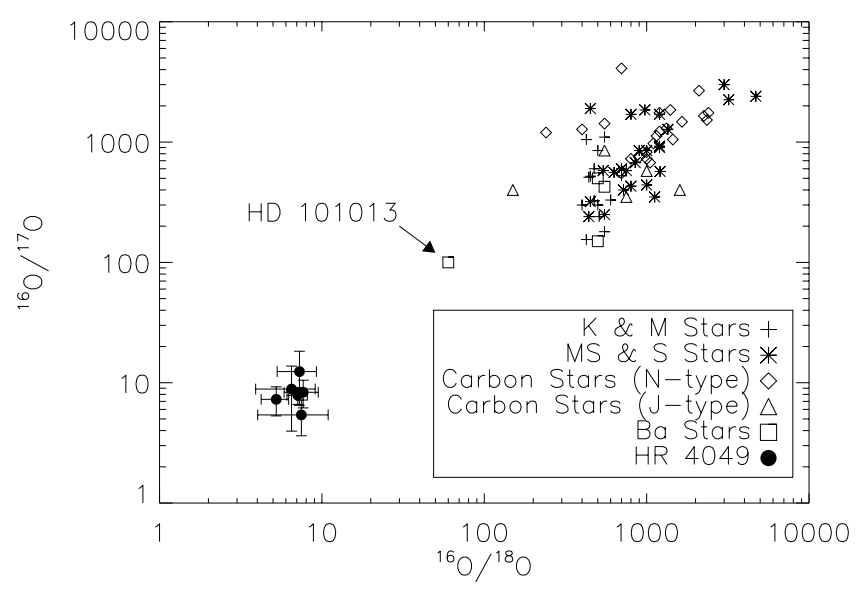

Fig. 2. Oxygen isotopic ratios for HR 4049 derived from the $\mathrm{CO}_{2}$ bands in the optically thin limit compared to measured isotopic ratios for RGB and AGB stars. Data are from: K \& M Stars (Harris \& Lambert 1984; Harris et al. 1988), MS \& S Stars (Harris et al. 1985b; Smith \& Lambert 1990), Carbon Stars (Harris et al. 1987), Ba Stars (Harris et al. 1985a)

of excited molecules to the total number of molecules $\mathcal{N}$ so that

$I_{\nu}=\mathcal{N} \frac{g_{u}}{\mathcal{P}(T)} \mathrm{e}^{-E_{u} / k T} A_{u l} h \nu$

with $g_{u}$ the statistical weight of the upper level, $\mathcal{P}(T)$ the partition function at temperature $T, E_{u}$ the energy of the upper level and $A_{u l}$ the Einstein coefficient for spontaneous emission. When considering the bands of two different isotopomers (labeled as 1 and 2), the corresponding molecular number ratios are thus given by

$$
\begin{aligned}
\frac{\mathcal{N}_{1}}{\mathcal{N}_{2}} & =\frac{I_{\nu, 1}}{I_{\nu, 2}} \frac{\mathcal{P}_{1}}{g_{u, 1}}\left(\frac{\mathcal{P}_{2}}{g_{u, 2}}\right)^{-1} \frac{A_{2}}{A_{1}} \frac{\nu_{2}}{\nu_{1}} \mathrm{e}^{-\left(E_{u, 2}-E_{u, 1}\right) / k T} \\
& \approx \frac{I_{\nu, 1}}{I_{\nu, 2}}
\end{aligned}
$$

When comparing the same ro-vibrational bands for different isotopomers, all terms except the intensity ratio are of order unity; even when comparing isotopomers with a different symmetry (e.g. ${ }^{12} \mathrm{C}^{16} \mathrm{O}_{2}$ and ${ }^{16} \mathrm{O}^{12} \mathrm{C}^{17} \mathrm{O}$ ) the terms $\mathcal{P} / g_{u}$ are typically only a factor of 2 . By combining Eqs. (2) and (4) one can then directly estimate the isotopic 
ratios from the observed line fluxes. As six isotopomers are observed in three different bands, each oxygen isotopic ratio can be independently determined six times from the values listed in Table 1 . It is straightforward to calculate the errors on the isotopic ratios from Eq. (4) adopting an error of typically 0.75 Jy on the peak fluxes (see Table 1 ). As can be seen from Fig. 2, the isotopic ratios derived for the different bands using different isotopomers are the same to within the errors which indicates that the approximations used are valid. Combining the six derived values for each isotopic ratio, we finally find that ${ }^{16} \mathrm{O} /{ }^{17} \mathrm{O}=8.3$ \pm 2.3 and ${ }^{16} \mathrm{O} /{ }^{18} \mathrm{O}=6.9 \pm 0.9$.

\section{Discussion}

As can be seen from Fig. 2, the relative circumbinary ${ }^{17} \mathrm{O}$ and ${ }^{18} \mathrm{O}$ abundances derived from the $\mathrm{CO}_{2}$ bands in HR 4049 are two orders of magnitude higher than the surface abundances measured for the bulk of the evolved stars. Although we cannot entirely rule out the possibility that ${ }^{16} \mathrm{O}$ depletion or ${ }^{17} \mathrm{O}$ or ${ }^{18} \mathrm{O}$ enrichment occurred in the circumbinary disk as a consequence of fractionation, this process seems unlikely given its low efficiency or the low temperatures at which it generally occurs. We therefore believe that the observed abundances reflect the abundances in the stellar wind (currently or in the past) of HR 4049. This conclusion is strengthened by an evolutionary link between HR 4049 and the Ba star HD 101013. As the mass lost by HR 4049 is not only stored in the circumbinary disk but also partly transferred to the (currently undetected) companion, the surface abundances of this companion would be the same as those found in the circumbinary disk if no mixing occurs. When mixing would occur, the relative ${ }^{17} \mathrm{O}$ and ${ }^{18} \mathrm{O}$ abundances at the surface of the companion star would both decrease, and follow a track in Fig. 2 which goes up and to the right, exactly toward the Ba star HD 101013. As the companion to HR 4049 will probably only be observable at the time it becomes a red giant, and given the time it would take the companion to reach this phase, there is plenty of time for mixing of this material and reach abundances similar to those of HD 101013 at the time it becomes an observable Ba star. Indeed, if mixing occurred in HD 101013 between the time where its companion dumped enriched material onto the surface and present day, the relative ${ }^{17} \mathrm{O}$ and ${ }^{18} \mathrm{O}$ abundances must have been higher in the past.

It is important to realize that HR 4049 has most likely gone through a phase of case $\mathrm{C}$ Roche lobe overflow. Most scenarios for case $\mathrm{C}$ Roche lobe overflow assume highly non-conservative mass transfer, where most of the material lost is stored in a circumbinary disk or lost from the system rather than being transferred to the companion star. If we assume that the observed $\mathrm{CO}_{2}$ is only formed from material lost during this phase, and furthermore that highly enriched material dumped onto the companion is diluted with $0.6 M_{\odot}$ (the total mass of the companion, see Bakker et al. 1998) of material having solar abundances, it would show the abundances observed in HD 101013 if it would have accreted no more than $\sim 0.06 M_{\odot}$; the remaining mass lost by the primary would then have to be dumped in the circumbinary disk. As a typical AGB envelope mass is $0.3-0.5 M_{\odot}$ this implies that almost the entire envelope must exhibit the extreme ${ }^{17} \mathrm{O}$ and ${ }^{18} \mathrm{O}$ enrichments we observe in the $\mathrm{CO}_{2}$ bands.

The relative enrichment of the oxygen isotopes measured here cannot be explained with current nucleosynthesis models for single stars. Some models might be stretched to explain either the ${ }^{17} \mathrm{O}$ or ${ }^{18} \mathrm{O}$ overabundance, but to our knowledge there is no model that can explain the simultaneous enrichment of both isotopes to the extent observed in HR 4049 (M. Lugaro, T. Blöcker, J. Lattanzio, private communication). It is likely that the binary nature is an important element for explaining these abundances.

Acknowledgements. We appreciate encouraging discussions with Rens Waters. J. C. acknowledges support from an NWO Pionier grant.

\section{References}

Bakker, E. J., Lambert, D. L., van Winckel, H., et al. 1998, A\&A, 336, 263

Bakker, E. J., van Dishoeck, E. F., Waters, L. B. F. M., \& Schoenmaker, T. 1997, A\&A, 323, 469

Beintema, D. A., van den Ancker, M., Molster, F. J., et al. 1996, A\&A, 315, L369

Cami, J., Justtanont, K., de Jong, T., et al. 1997, in First ISO workshop on Analytical Spectroscopy, ed. A. M. Heras, K. Leech, N. R. Trams, \& M. Perry, ESA-SP419, 159

Cami, J., Yamamura, I., de Jong, T., Tielens, A. G. G. M, \& Waters, L. B. F. M. 2000, A\&A, 360, 562

Cami, J., et al. 2001, A\&A, in preparation (Paper I)

Geballe, T., Noll, K., Whittet, D., \& Waters, L. B. F. M. 1989, ApJ, 340, L29

Guillois, O., Ledoux, G., \& Reynaud, C. 1999, ApJ, 521, L133

Harris, M. J., \& Lambert, D. L. 1984, ApJ, 285, 674

Harris, M. J., Lambert, D. L., Hinkle, K. H., et al. 1987, ApJ, 316,294

Harris, M. J., Lambert, D. L., \& Smith, V. V. 1985a, ApJ, 292, 620

Harris, M. J., Lambert, D. L., \& Smith, V. V. 1985b, ApJ, 299, 375

Harris, M. J., Lambert, D. L., \& Smith, V. V. 1988, ApJ, 325, 768

Justtanont, K., Feuchtgruber, H., de Jong, T., et al. 1998, A\&A, 330, L17

Markwick, A., \& Millar, T. 2000, A\&A, 359, 1162

Ryde, N., Eriksson, K., Gustafsson, B., Lindqvist, M., \& Olofsson, H. 1998, Ap\&SS, 255, 301

Smith, V. V., \& Lambert, D. L. 1990, ApJS, 72, 387

van Winckel, H., Waelkens, C., \& Waters, L. B. F. M. 1995, A\&A, 293, L25

Waters, L. B. F. M., Lamers, H. J. G. L. M., Snow, T. P., et al. 1989, A\&A, 211, 208

Waters, L. B. F. M., Trams, N. R., \& Waelkens, C. 1992, A\&A, 262, L37 